\title{
Correction to: Coverage-Oriented Diversification of Keyword Search Results on Graphs
}

Ming Zhong, Ying Wang, and Yuanyuan Zhu

\section{Correction to:}

Chapter "Coverage-Oriented Diversification of Keyword

Search Results on Graphs" in: J. Pei et al. (Eds.):

Database Systems for Advanced Applications,

LNCS 10828, https://doi.org/10.1007/978-3-319-91458-9_10

In the originally published version of chapter 10 the funding information in the acknowledgement section was incomplete. This has now been corrected. 